Ergod. Th. \& Dynam. Sys. (1988), 8, 621-632

Printed in Great Britain

\title{
及-Stability is not dense in Axiom A
}

\author{
S. E. PATTERSON* \\ Northwestern University, Evanston, Illinois, USA
}

(Received 8 October 1986 and revised 15 July 1987)

\begin{abstract}
An example of a diffeomorphism $f$ on a three dimensional manifold $M^{3}$ is constructed so that $f$ satisfies Axiom A, has a cycle and $f$ has a neighborhood $N$ in $\operatorname{Diff}^{r}\left(M^{3}\right)$ so that each $g$ in $N$ is not $\Omega$-stable. Existing techniques allow one to extend this example to any compact manifold of dimension greater than two.
\end{abstract}

\section{Introduction}

We are concerned here with the following question: can every Axiom A diffeomorphism of a compact $n$-dimensional manifold $M^{n}$ be approximated by an $\Omega$-stable one? Palis's work on $\Omega$-explosions [5] and Smale's $\Omega$-stability theorem [9] reduce this question to: if $f$ satisfies Axiom $\mathrm{A}$ and has a cycle, can $f$ be approximated by an $\Omega$-stable diffeomorphism? The answer turns out to depend upon the dimension $n$ of $M^{n}$. For $n=1$ a diffeomorphism satisfies Axiom $A$ if and only if it is $\Omega$-stable (using Peixoto [7]). For $n=2$ Newhouse and Palis [2] show that the question has an affirmative answer. In fact they prove a stronger result.

Theorem [Newhouse and Palis]: if $f$ is an Axiom A diffeomorphism of a compact two dimensional manifold with non-wandering set $\Omega(f)$ then $f$ may be approximated by an $\Omega$-stable $\mathrm{g}$ such that $\Omega(\mathrm{g})=\Omega(f)$.

This theorem is proved by showing that to every two dimensional Axiom A system with a cycle there may be applied a prescribed perturbation which leaves the non-wandering set unchanged while 'breaking' the cycle connections. After finitely many such perturbations the new system is Axiom $A$ ( $\Omega$ is unchanged) and has no cycles so it is $\Omega$ stable. The fact that $\Omega(g)=\Omega(f)$ in this case is more than a corollary to the proof that our question has a positive answer in dimension two. It is an essential feature of the prescribed perturbation. That this type of perturbation does not extend to higher dimensions is demonstrated by a pair of examples due to Pugh, Walker, and Wilson [8] and Dankner [1]. These are examples of Axiom A systems (a flow and a diffeomorphism respectively) in dimension three with finite nonwandering sets and cycles such that any attempt to 'break' the cycle creates homoclinic points and $\Omega$-havoc. Consequently, these systems cannot be approximated by $\mathbf{\Omega}$-stable systems with the same nonwandering set-as the original system. This still

* Current Address: Department of Mathematics and Computer Science, Carleton College, Northfield, MN 55057, USA. 
does not answer our original question, however. A generalization of [6] shows that Dankner's system does indeed have $\Omega$-stable approximations albeit with much enlarged nonwandering sets. It would seem plausible, therefore, that our question has an affirmative answer for every dimension.

It is the purpose of this paper to prove the following

THEOREM. In every isotopy class of Diff $\left(M^{n}\right), n \geq 3$ there is a diffeomorphism satisfying Axiom A which cannot be $C^{2}$ approximated by an $\Omega$-stable diffeomorphism.

This is a strengthening of a theorem of Dankner [1] which indicates that the diffeomorphism cannot be approxiated by an $\Omega$-stable diffeomorphism with the same nonwandering set as the original. We will modify Dankner's example to produce a diffeomorphism $F$ with the following properties:

(1) $F$ satisfies Axiom A

(2) $F$ has cycles

(3) Every diffeomorphism, $G, C^{2}$ near $F$ either has

(a) cycles

or

(b) a wild hyperbolic set,

so $G$ is not $\Omega$-stable.

2.

We now construct a two dimensional prototype of the example. We will construct an Axiom A diffeomorphism $f$ of the 2-sphere which has a cycle and consider perturbations of $f$ with certain restrictions on the type of perturbation to be made.

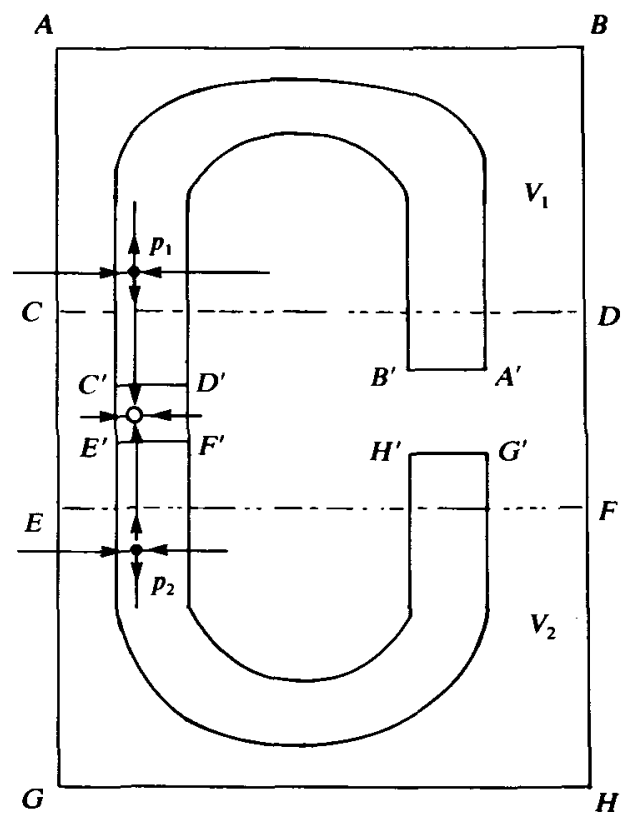

FIGURE 1 
In higher dimensions, the example may be constructed so that these restrictions are unnecessary. Given these restrictions we will see that any system 'near' $f$ either has a cycle or a wild hyperbolic set. Thus any perturbation of $f$ (modulo the restrictions) is not $\boldsymbol{\Omega}$-stable.

Let $V$ be a closed rectangle and define $f_{0}: V \rightarrow V$ so that $V$ contains two horseshoes and one sink as shown in figure 1. Let $V_{1}$ and $V_{2}$ be the subrectangles shown and define $\Lambda_{i}=\bigcap_{n \in Z} f^{n} V_{i}$ for $i=1,2$. Let $p_{i}$ be the fixed point in $V_{i}$ with positive eigenvalues for $i=1,2 . \Lambda_{1}$ and $\Lambda_{2}$ are non-trivial, zero-dimensional, hyperbolic basic sets. These basic sets may be constructed with arbitrarily large stable and unstable thicknesses. In particular we may assume $\tau^{u}\left(\Lambda_{2}\right) \cdot \tau^{s}\left(\Lambda_{1}\right)>1$. See [4] for definitions and discussion.

We now modify $f_{0} \mid V$ by pushing some of $W^{u}\left(\Lambda_{2}\right)$ so that it meets some of $W^{s}\left(\Lambda_{1}\right)$ away from $\Lambda_{1}$ and $\Lambda_{2}$ as shown in figure 2. Call the resulting map $f_{1}$.

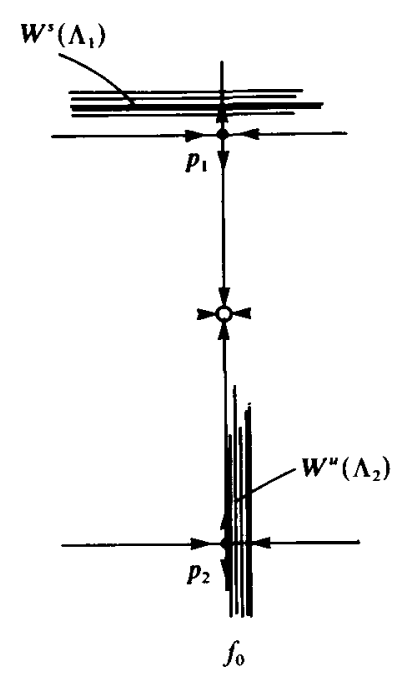

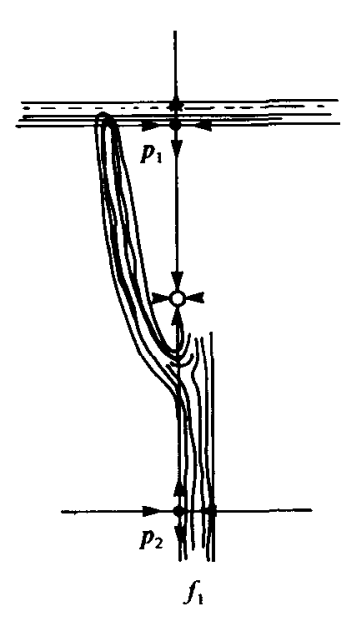

FIGURE 2

Since $\tau^{u}\left(\Lambda_{2}\right) \cdot \tau^{s}\left(\Lambda_{1}\right)>1$, this can be done so that $C^{2}$ persistent tangencies between $W^{u}\left(\Lambda_{2}\right)$ and $W^{s}\left(\Lambda_{1}\right)$ are created. It is clear that no new recurrence is created.

Now extend $f_{1}$ to $f_{2} \in \operatorname{Diff}\left(S^{2}\right)$ defined by figure 3 .

Further modify $f_{2}$ to $f_{3}$ by composing $f_{2}$ with a diffeomorphism supported on the rectangle $R$ of figure 3 so that $W^{u}\left(\Lambda_{1}, f_{3}\right) \pitchfork W^{s}(p) \neq \varnothing$. Call the resulting diffeomorphism $f$.

It is important to observe that those points of tangency between $W^{u}\left(\Lambda_{2}\right)$ and $W^{s}\left(\Lambda_{1}\right)$ are still not in the limit set. They are not even non-wandering. This is a result of the construction of the part of the cycle connecting $p$. to $q$. To see this, consider any point in some neighborhood of a point-of tangency. The only way this point can return to this neighborhood is to pass along the cycle and reenter $V$ but any point which leaves $V$ does so through $R$ and is above $W^{u}(p)$ so is in the stable 


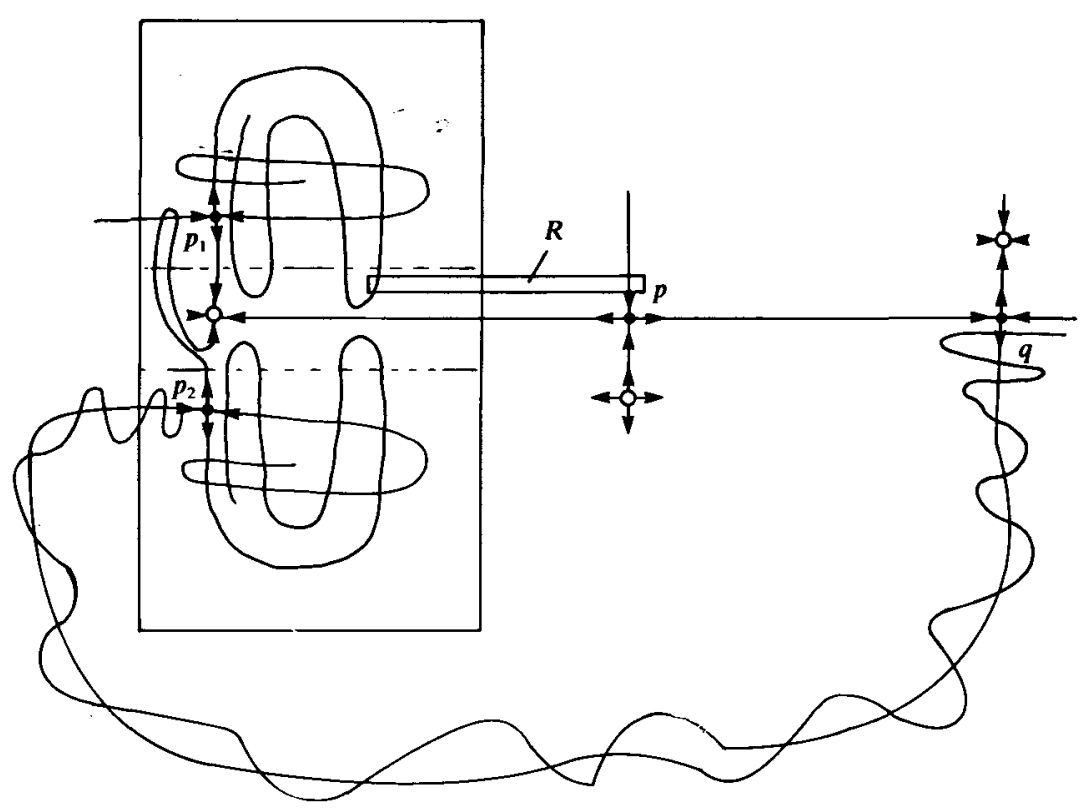

FIGURE 3

manifold of either $p$ or one of the sinks. We conclude that the points of tangency are wandering and $f$ has a hyperbolic limit set and a cycle, so $f$ is not $\Omega$-stable.

Because this is an example in two dimensions we could break the cycle according to Newhouse and Palis [2]. This would be accomplished by pushing a fundamental domain of $W^{u}(p)$ in the coincidence $W^{u}(p) \cap W^{s}(q)$ upward so that $W^{u}(p) \backslash\{p\}$ is completely contained in the stable manifolds of the sinks. In order to construct our two dimensional example, however, we make the following restriction on the type of perturbation to be allowed. This restriction is unnecessary in higher dimensions.

Restriction. Any perturbation which breaks the coincidence pushes some of $W^{u}(p)$ below $W^{s}(q)$.

Now any system $g$ near $f$ either leaves the coincidence intact and thus has a cycle and is therefore $\Omega$-unstable or has transverse intersections between $W^{s}\left(\Lambda_{2}\right)$ and $W^{u}\left(\Lambda_{1}\right)$. This is because these sets accumulate below and above the coincidence respectively and we are assuming that any perturbations of the coincidence pushes some of $W^{u}(p)$ below $W^{s}(q)$. It follows from Lemma 8 of [3] that $g$ has a wild hyperbolic set.

We conclude that the diffeomorphism $f$ is isolated from the $\Omega$-stable systems since any perturbation (given the restrictions) of $f$ either has a cycle or a wild hyperbolic set. We point out, however, that while $L(f)$ is hyperbolic, $f$ does not satisfy Axiom A. This is because $W^{u}(p) \cap W^{s}(q) \subset \Omega$ but $W^{u}(p) \cap W^{s}(q) \cap L=\varnothing$. To construct an Axiom A example one merely needs to connect two $p-q$ coincidences in tandem as shown in figure 4. 


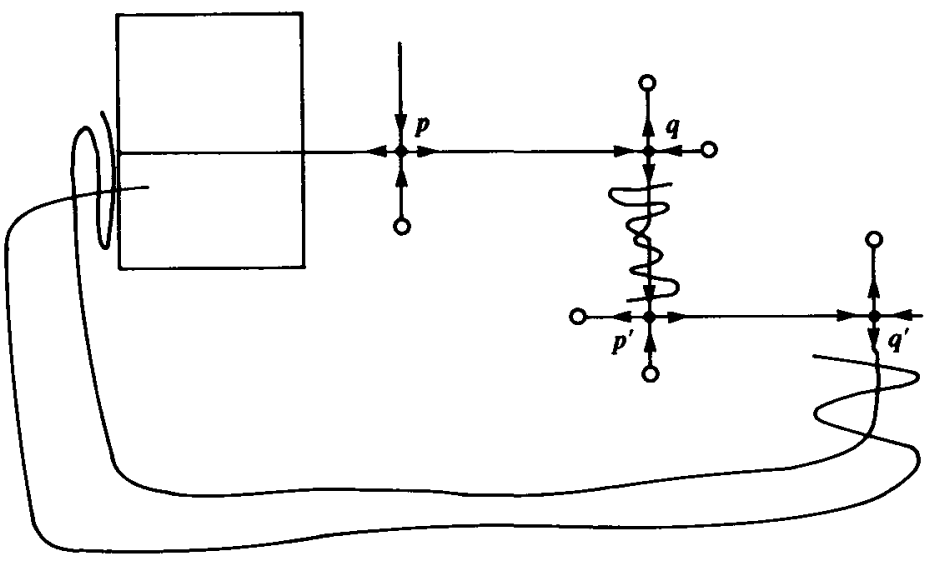

FIGURE 4

We also extend the restrictions on breaking the coincidence $p-q$ to include $p^{\prime}-q^{\prime}$. It is straightforward to verify that the resulting system is Axiom $A$ and is isolated from the $\Omega$-stable systems given these restrictions.

3.

In this section we examine a local example due to Dankner [1] and in $\$ 4$ we show how to modify it to produce a local example with the desired characteristics, namely, an Axiom $A$ system which cannot be approximated by an $\Omega$-stable system. The theorem will follow immediately from the proof of Theorem 6.1 in [1] which shows that therefore each isotopy class of Diff $\left(M^{n}\right), n \geq 3$ contains such a system. Only a sketch of Dankner's example appears here. The reader is encouraged to see [1] for the details. We will follow the notation in that paper.

A two dimensional analogue of Dankner's three dimensional system is shown in figure 5 where we assume the same coincidence breaking restrictions as in $\S 2$.

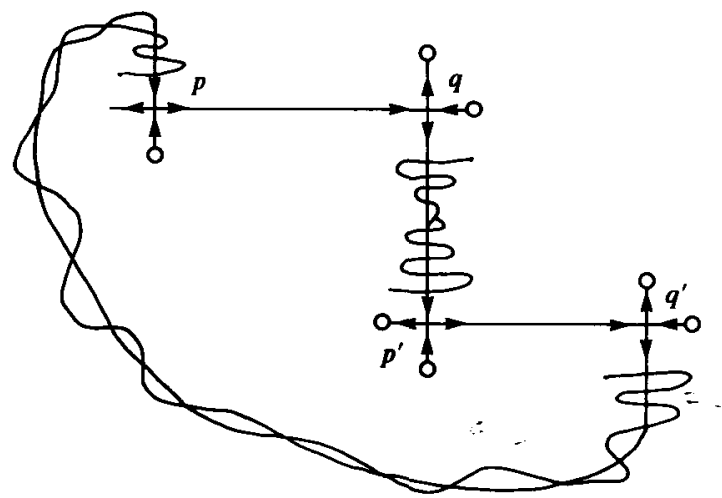

FiguRE 5 
The essential feature of this system is that any perturbation of this system which breaks the coincident cycle connections (given the restrictions) creates homoclinic points and hence $\Omega_{-}$-explosions. Since the coincidences are the only breakable cycle connections and since the original system has a finite non-wandering set one may conclude that, given these restrictions, this Axiom A system cannot be approximated by an $\Omega$-stable system with the same nonwandering set.

Dankner's three dimensional example, of course, does not require the restrictions on breaking the coincidence to reach the same conclusion. His example begins with two identical open subsets $U$ and $U^{\prime}$ in $\mathbb{R}^{3}$ with $F_{0}: U \cup U^{\prime} \rightarrow \mathbb{R}^{3}$ defined similarly on each and shown for $U$ in figure 6.

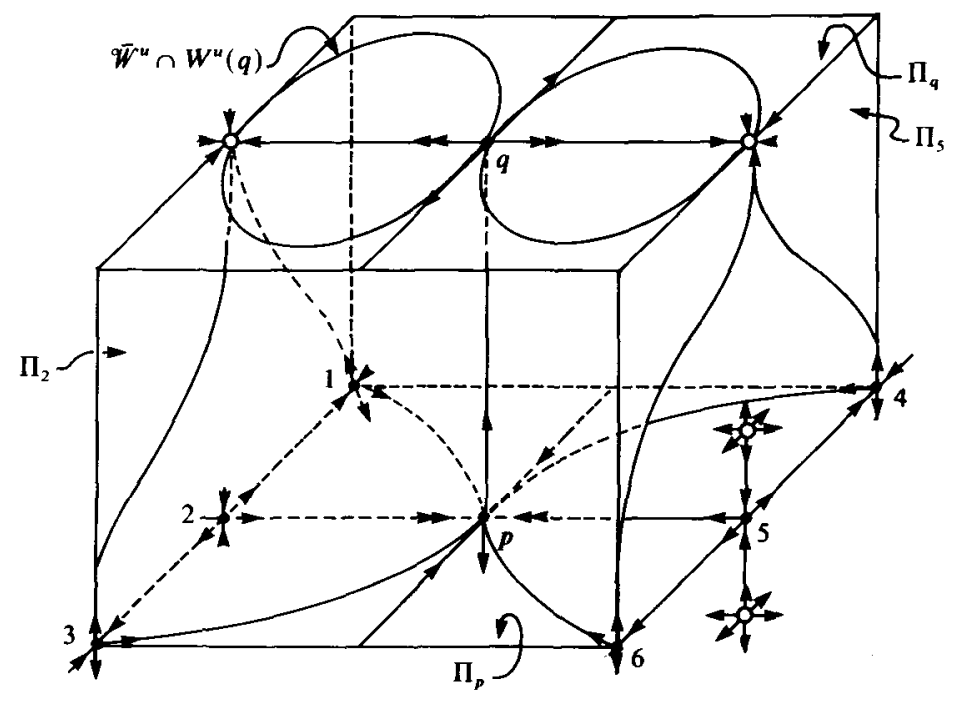

FIGURE 6

The figure shown is in the interior of $U$. The planes $\Pi_{q}, \Pi_{p}, \Pi_{2}, \Pi_{5}$ and the plane $\Pi_{*}$ through $p$ and $q$ parallel to $\Pi_{2}$ are all locally invariant under $F_{0}$. Furthermore $\Pi_{*}$ is a plane of symmetry. The points $1,2,3,4,5,6, p, q$ and all sources and sinks are hyperbolic fixed points. We note that there are other fixed points in $U$ which are not shown as we are not concerned with them here. The saddle points $1,2,3$, $4,5,6$ and $q$ all have two dimensional unstable manifolds whereas $p$ has a one dimensional unstable manifold. Let $\mu, \lambda_{1}$ and $\lambda_{2}$ with $0<\mu<1<\lambda_{1}<\lambda_{2}$ be the eigenvalues of $T F_{0}$ at $q$. In this case $\lambda_{2}^{-1}, \lambda_{1}^{-1}$ and $\mu^{-1}$ are the eigenvalues of $T F_{0}$ at $p$.

Let $W^{u}=W^{u}(1) \cup W^{u}(3) \cup W^{u}(4) \cup W^{u}(6) \cup W^{u}(p) . W^{u}-W^{u}(p)$ is the union of two dimensional unstable manifolds. Let $\mathscr{X}=\mathscr{W}^{u} \cap W^{s}(p)$. Near $p$, $\mathscr{W}^{u}$ consists of vertical one dimensional fibers over $\mathscr{X}$. We assume that $F_{0}$ has sufficient symmetry that near $q, \overline{W^{u}} \cap W^{u}(q)$ is the image under vertical projection of $\mathscr{X}$ into $W^{u}(q)$. Denote the corresponding structures in $U^{\prime}$ with primes. So the fixed points of $F_{0} \mid U^{\prime}$ are $1^{\prime}, 2^{\prime}, 3^{\prime}$ etc. We assume that $\left(U \cup F_{0}(U)\right) \cap\left(U^{\prime} \cup F_{0}\left(U^{\prime}\right)\right)=\varnothing . F$ is an extension of $F_{0}$ to all of $\mathbb{R}^{3}$ and $\hat{F}$ is defined to be $F$ composed with sixteen diffeomorphisms 
$f_{1}, \ldots, f_{16}$ of $\mathbb{R}^{3}$ each of which is supported on a thin cylinder. The effect of these $f_{i}$ 's is to push a piece of $W^{u}(q)$ (resp. $W^{u}\left(q^{\prime}\right)$ ) into transversal intersection with one component of $W^{s}\left(2^{\prime}\right) \backslash\left\{2^{\prime}\right\}$ or $W^{s}\left(5^{\prime}\right) \backslash\left\{5^{\prime}\right\}\left(\right.$ resp. $W^{s}(2) \backslash\{2\}$ or $\left.W^{s}(5) \backslash\{5\}\right)$. This is done carefully in [1] so that one sees that no new recurrence is created. $\hat{F}$ is an Axiom A diffeomorphism with a finite non-wandering set and has cycles. The cycles in $\Omega(\hat{F})$ may be represented by the following diagram.

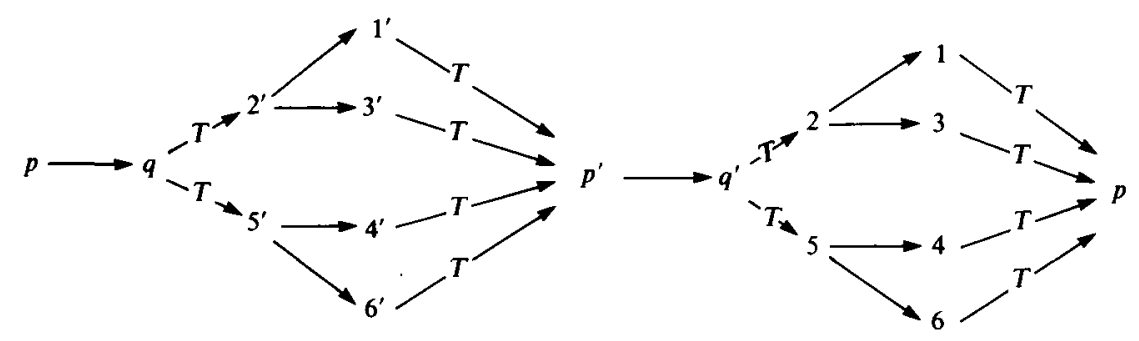

Figure 7

Here $\delta \rightarrow \beta$ means $W^{u}(\delta) \cap W^{s}(\beta) \neq \varnothing$ and $\delta \mp \beta$ means $W^{u}(\delta) \hbar W^{s}(\beta) \neq \varnothing$. In any cycle of $\hat{F}$ there are only two breakable (non-transverse) types of cycle connections:

1. $p \rightarrow q$ (and $p^{\prime} \rightarrow q^{\prime}$ )

and

2. $2 \rightarrow 1$ (and $2^{\prime} \rightarrow 1^{\prime}, 2 \rightarrow 3,2^{\prime} \rightarrow 3^{\prime}, 5^{\prime} \rightarrow 4^{\prime}, 5^{\prime} \rightarrow 6^{\prime}, 5 \rightarrow 4$ and $5 \rightarrow 6$ ).

We first consider type 2 . It is easily seen that due to transversality of $W^{\prime \prime}\left(q^{\prime}\right)$ with $W^{s}(2)$ and hyperbolicity of 2 that, for diffeomorphisms near $\hat{F}$, if $W^{u}(2) \cap W^{s}(1)=\varnothing$ then $W^{u}\left(q^{\prime}\right) \cap W^{s}(1) \neq \varnothing$.

In fact we simplify the above construction by dispensing with those eight cylinders $f_{2}, f_{4}, f_{6}, \ldots, f_{16}$ which connect $W^{u}(q)$ and $W^{u}\left(q^{\prime}\right)$ to the components of $W^{s}\left(2^{\prime}\right)$,

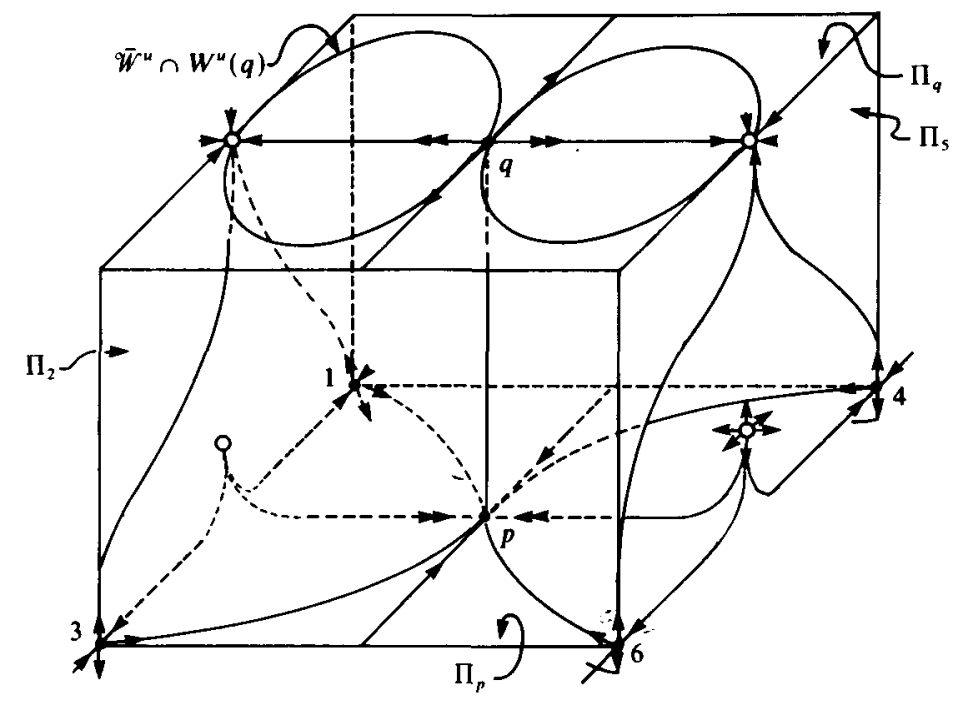

Figure 8 
$W^{s}\left(5^{\prime}\right), W^{s}(2)$ and $W^{s}(5)$ which are below $\Pi_{p^{\prime}}$ and $\Pi_{p}$ and then perturbing $W^{u}(2)$, $W^{u}(5), W^{u}\left(2^{\prime}\right)$ and $W^{u}\left(5^{\prime}\right)$ downward. The resulting picture in $U$ is shown in figure 8. (The 'fingers' of $W^{u}\left(q^{\prime}\right)$ pushed along the cylinders are not shown to simplify the figure.) This simplified system has the same essential properties of Dankner's original. It has the same non-wandering set so it too is Axiom $A$ and it has unbreakable cycles as we will see.

The resulting diagram is then

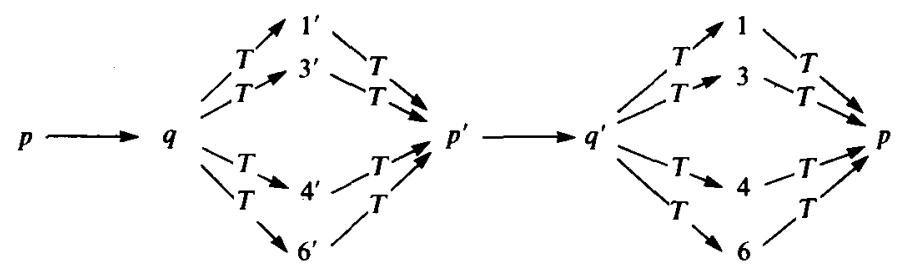

Figure 9

and the only type of breakable cycle connection is $p \rightarrow q$. We will hereafter assume that $\hat{P}$ is so defined.

Remark 3.6. One may further simplify the construction by dispensing with $U^{\prime}$ and connecting $W^{u}(q)$ with $W^{s}(2)$ and $W^{u}(5)$ from above $\Pi_{p}$. The resulting system while not Axiom A (all of $W^{u}(p) \cap W^{s}(q)$ is non-wandering) does have a hyperbolic limit set and fully retains all of the essential perturbation behaviour of the original example. This is an example of an $L$-hyperbolic system which cannot be approximated by an $\Omega$-stable (or $L$-stable) system with the same limit set.

We return to $\hat{F}$ and consideration of the only (pair of) breakable cycle connection(s): $p \rightarrow q$ (and $p^{\prime} \rightarrow q^{\prime}$ ). The essential feature of this coincident cycle connection is the following.

Let $I \subset W^{u}(p) \cap W^{s}(q)$ be a closed interval, then there is a neighborhood $N$ of $I$ such that (see figure 10)

1. $N \backslash\left(W^{u} \cap N\right)$ has four components $R_{1}, R_{2}, R_{3}$ and $R_{4}$

2. $W^{u}\left(q^{\prime}\right)$ accumulates on $W^{u} \cap N$ from $R_{2}$ and $R_{4}$

3. $W^{s}\left(\alpha^{\prime}\right)$ accumulates on $W^{u} \cap N$ from $R_{1}$ and $R_{3}$ for $\alpha^{\prime}=1^{\prime}, 3^{\prime}, 4^{\prime}, 6^{\prime}$

4. $W^{u}\left(q^{\prime}\right) \cap W^{s}\left(\alpha^{\prime}\right)=\varnothing$ for $\alpha^{\prime}=1^{\prime}, 3^{\prime}, 4^{\prime}, 6^{\prime}$.

We can now see that this part of the construction from $F$ to $\hat{F}$ introduces no new nonwandering points since such points would have nearby orbits which would pass from $U^{\prime}$ through $U$ and back to $U^{\prime}$ (or vice-versa). But this is impossible. Any such orbit entering $U$ must do so through one of the cylinders from $U^{\prime}$ to $U$ thus the orbit passes through the neighborhood $N$ in regions $R_{2}$ or $R_{4}$. These regions are completely contained in the stable manifolds of sinks in $U$, however, so the orbit cannot return to $U^{\prime}$. Likewise, orbits which do travel from $U$ to $U^{\prime}$ have points in the regions $R_{1}$ or $R_{3}$ so $W^{u}$ acts as a barrier preventing orbits which pass from $U^{\prime}$ to $U$ from returning to $U^{\prime}$. Thus we have property 4 and we may conclude that $\Omega(F)$ is finite.

This barrier property of $\mathscr{W}^{u}$ is delicate and depends on the coincidence of $W^{u}(p)$ and $W^{s}(q)$. Properties 1-3 guarantee that if this coincidence and the corresponding 


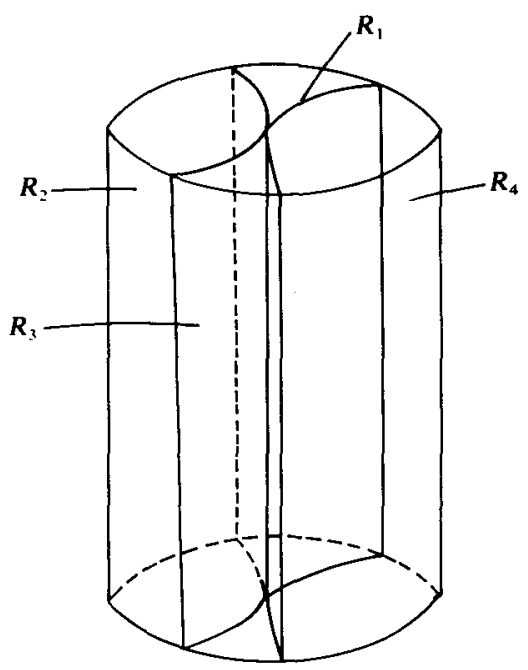

FIGURE 10

coincidence in $U^{\prime}$ are broken for some $g$ near $F$ then $g$ will have transverse homoclinic points and thus $\Omega(g)$ will be infinite.

4.

In this section we see how to modify the example of $\$ 3$ to create an Axiom $A$ system without $\Omega$-stable perturbations.

Let $f_{1}: V \rightarrow V$ be the two dimensional system described in $\S 2$ and shown in figures 1 and 2 . Recall that $f_{1}$ has persistent tangencies between $W^{u}\left(\Lambda_{2}\right)$ and $W^{s}\left(\Lambda_{1}\right)$.

Let $I=[-1,1]$ and define

$$
\tilde{f}=f_{1} \times h: V \times I \rightarrow \mathbb{R}^{3},
$$

where $h(x)=\lambda x$ with $\lambda>1$ larger than the largest eigenvalue of $T f_{1}$ on $V$. Thus $f: V \rightarrow \mathbb{R}^{2}$ is normally hyperbolically imbedded in $\tilde{f}: V \times I \rightarrow \mathbb{R}^{3}$ and $\tilde{\Lambda}_{1}=\Lambda_{1} \times\{0\}$ and $\tilde{\Lambda}_{2}=\Lambda_{2} \times\{0\}$ are basic sets for $\tilde{f}$ with two dimensional unstable manifolds. Furthermore $W^{u}\left(\tilde{\Lambda}_{2}\right)$ has persistent tangencies with $W^{s}\left(\tilde{\Lambda}_{1}\right)$.

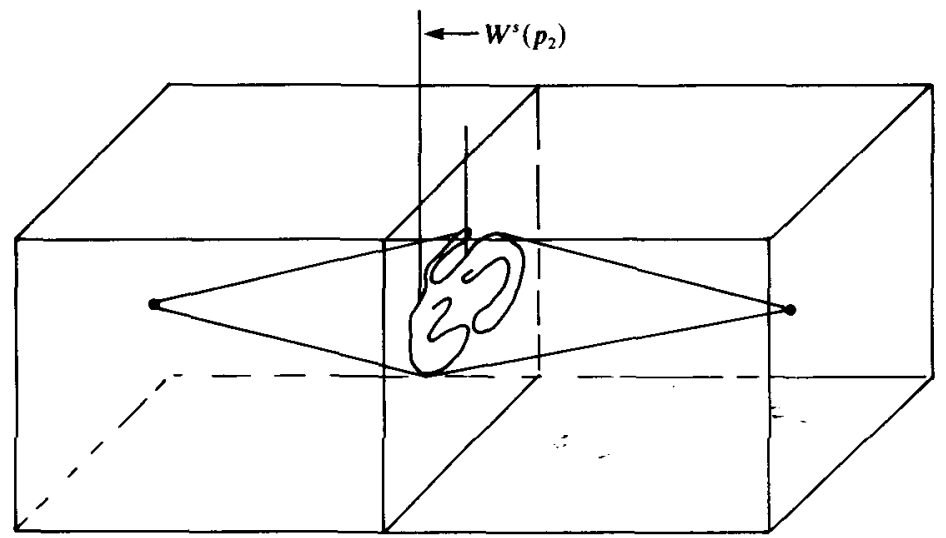

FIGURE 11 


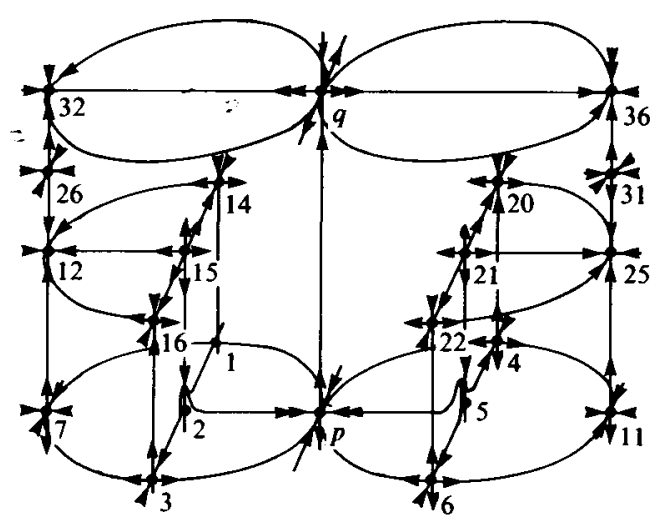

Figure 12

Let $J$ be a closed interval containing $I$ in its interior, let $\hat{V}=V \times J$ and extend $\tilde{f}$ to $\hat{f}: \hat{V} \rightarrow \hat{V}$ by giving $\hat{f}$ a sink in each component of $V \times(J \backslash I)$. See figure 11 .

We are now in a position to define our example. Let $\hat{F}$ be the system described in $\S 3$. Let $F_{1}=\mathbb{R}^{3} \rightarrow \mathbb{R}^{3}$ agree with $\hat{F}$ outside the union of two small balls containing the sinks 12 and 25 (see figure 12). Inside these balls modify $\hat{F}$ so that one copy of $\hat{f}: \hat{V} \rightarrow \hat{V}$ is in each as shown in figure 13. Call these balls ${ }_{12} V$ and ${ }_{25} V$ and name the structures in them accordingly - i.e. ${ }_{12} \Lambda_{2}$ and ${ }_{12} \Lambda_{1}$ are the non-trivial basic sets in ${ }_{12} V$ etc. We maintain the symmetry of $\hat{F}$ so that ${ }_{12} V$ and its associated structures are the mirror image of ${ }_{25} V$ and its structures. As we describe those structures for ${ }_{25} V$ below it is to be understood that the structures for ${ }_{12} V$ are identical.

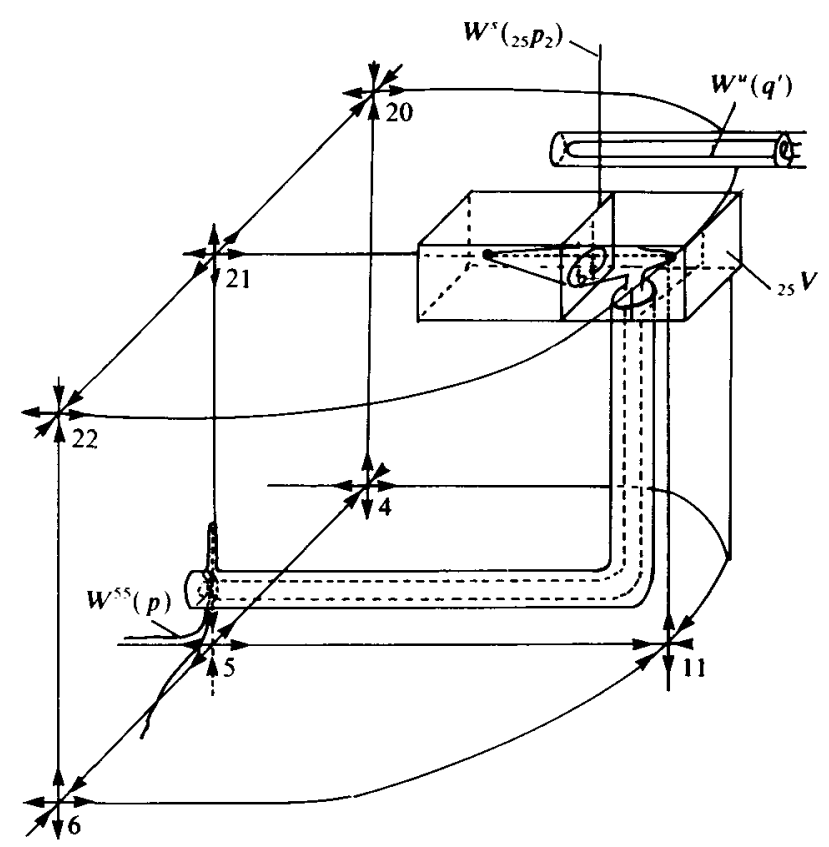

FiguRE 13 
Now modify the diffeomorphisms $f_{11}$ and $f_{15}$ which push pieces of $W^{u}\left(q^{\prime}\right)$ along thin cylinders so that $W^{u}\left(q^{\prime}\right)$ meets $W^{s}\left({ }_{25} p_{2}\right)$ transversely instead of $W^{s}(5)$. Finally push a piece of $W^{u}\left({ }_{25} \Lambda_{1}\right)$, along a thin cylinder, into transverse intersection with $W^{s}(5)$ as shown in figure 13. Call the resulting diffeomorphism $F$.

It is critical to our analysis that all of this be done so that

$$
\left[W^{u}\left({ }_{25} \Lambda_{1}\right) \cup W^{u}\left({ }_{25} \Lambda_{2}\right)\right] \cap W^{s}\left({ }_{25} p_{1}\right)=\varnothing .
$$

We rely upon this fact to conclude that those points of persistent tangency between $W^{u}\left({ }_{25} \Lambda_{2}\right)$ and $W^{s}\left({ }_{25} \Lambda_{1}\right)$ are wandering. One can see that $F$ has the following diagram:

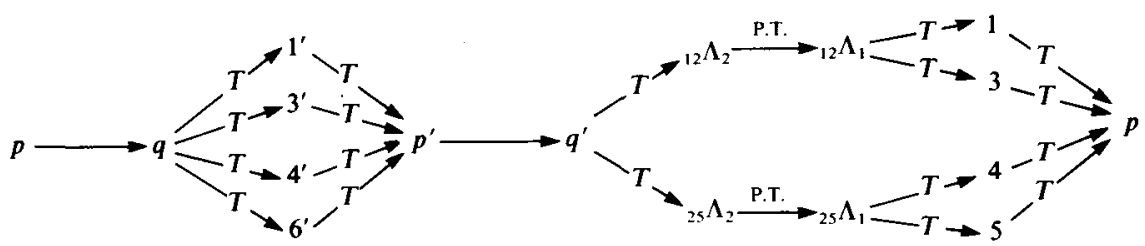

Figure 14

where ${ }_{25} \Lambda_{2} \rightarrow{ }_{25}^{P . T} \Lambda_{1}$ means that $W^{u}\left({ }_{25} \Lambda_{2}\right)$ has tangencies with $W^{s}\left({ }_{25} \Lambda_{1}\right)$ in a persistent way.

Let $G$ be any diffeomorphism $C^{2}$ near $F$. There are three cases:

(1) $G$ has both connections $p \rightarrow q$ and $p^{\prime} \rightarrow q^{\prime}$ intact, in which case $G$ has cycles and is not $\Omega$-stable.

(2) $G$ has only one of the connections, say $p \rightarrow q$, intact so

$$
W^{u}(q) \pitchfork W^{s}(p) \neq \varnothing
$$

In this case each $x \in W^{u}(p) \cap W^{s}(q)$ is non-wandering so $G$ is not $\Omega$-stable.

(3) $G$ has both connections broken in which case

$$
\left[W^{u}\left({ }_{25} \Lambda_{1}\right) \cup W^{u}\left({ }_{12} \Lambda_{1}\right)\right] \dagger W^{s}(q) \neq \varnothing .
$$

Assume without loss of generality that

$$
W^{u}\left({ }_{25} \Lambda_{1}\right) \hbar W^{s}(q) \neq \varnothing .
$$

Since the coincidence $p^{\prime} \rightarrow q^{\prime}$ is broken,

$$
W^{u}(q) \pitchfork W^{s}\left(q^{\prime}\right) \neq \varnothing .
$$

We conclude that

$$
W^{u}\left({ }_{25} \Lambda_{1}\right) \pitchfork W^{s}\left({ }_{25} \Lambda_{2}\right) \neq \varnothing
$$

while $W^{u}\left({ }_{25} \Lambda_{2}\right)$ has persistent tangencies with $W^{s}\left({ }_{25} \Lambda_{1}\right)$. From Lemma 8 of [3] we conclude that $G$ has wild hyperbolic set and thus is not $\Omega$-stable.

The methods of $\S 6$ of [1] extend this example to every isotopy class of $\operatorname{Diff}^{r}\left(M^{n}\right)$ for $n \geq 3$ and the theorem is proved.

Remark 4.5. The preceding discussion shows that $\Omega$-stability is not a $C^{2}$ dense property in Axiom A. The corresponding question for $C^{1}$ perturbations is unanswered. In a recent conversation with $S$. Newhouse it was pointed out that standard examples of systems which are $\Omega$-stable but not structurally stable have $C^{1}$ persistent 
tangencies and that perhaps these tangencies could be embedded into the cycle constructed above to yield an Axiom A system with no $C^{1}$ perturbations possessing the $\Omega$-stable property.

\section{REFERENCES}

[1] A. Dankner. Axiom A dynamical systems, cycles, and stability. Topology 19 (1980), 163-177.

[2] S. Newhouse \& J. Palis. Hyperbolic Non-Wandering Sets on Two-Dimensional Manifolds, Dynamical Systems, ed. M. Peixoto, Academic Press: New York, 1973, 293-301.

[3] S. Newhouse. The abundance of wild hyperbolic sets and non-smooth stable sets for diffeomorphisms. Publ. Math. IHES 50 (1979), 101-151.

[4] S. Newhouse. Lectures on Dynamical Systems, Progress in Math. 8, Birkhauser: 1980, Boston, 1-114.

[5] J. Palis. A Note on $\Omega$-Stability, Global Analysis eds S. S. Chern \& S. Smale, Amer. Math. Soc.: Providence, RI, 1970, 221-222.

[6] S. Patterson. Thesis, University of North Carolina (1982).

[7] M. Peixoto. Structural stability on two-dimensional manifolds. Topology 1 (1962).

[8] C. Pugh, R. Walker \& W. Wilson. On Morse-Smale Approximation - a Counter Example, J. Differential Equations 23 (1977), 173-182.

[9] S. Smale. The $\Omega$-Stability Theorem, Proc. Symp. in Pure Math. 14 (1970), 289-298. 